\title{
Application of Fuzzy Logic in the Detection of Eating Disorder
}

\author{
Ekholuenetale Michael ${ }^{1}$ \\ ${ }^{1}$ Women's Health and Action Research Centre, Km11, Lagos-Benin Expressway, Igue-Iheya, Benin City. \\ Nigeria.
}

\begin{abstract}
Eating disorders are critical and often fatal illnesses that cause severe disturbances. Fixation with food, body weight, and shape may also signal an eating disorder. Eating disorders are mental disorders identified by abnormal eating habits that negatively affect a person's physical or mental health. Despite the troubles that arise from various key eating disorders, many care givers and nutritionists have not bothered on mechanisms to measure these habits as to correct it and avert its ugly consequences. It is in the light of the above that I applied fuzzy logic, a form of many-valued logic in which the truth values of variables may be any real number between 0 and 1, considered to be "fuzzy", which by contrast, in Boolean logic, the truth values of variables may only be 0 or 1, often called "crisp" values. Fuzzy logic was employed to handle the concept of partial truth, where the truth value may range between completely true and completely false in the design and implementation of eating disorder diagnosis system for the detection of eating disorder using fuzzy logic a branch of soft computing.
\end{abstract}

Key words: Fuzzy logic, Probability, Disorder, Soft computing, Classifier

\section{Background to the Study}

An individual has an eating disorder when his attitude to food, weight, body size or shape lead to marked changes in their eating or exercise behaviours, which interfere with their life and relationships. Eating and exercise behaviours that people with eating disorders may engage in include: dieting, fasting, overexercising, using slimming pills, diuretics, laxatives, vomiting, or binge eating (consumption of an unusually large amount of food accompanied by a sense of loss of control). Eating disorders are not just about food and weight. They are also not about vanity or will-power. Eating disorders are serious and potentially life threatening mental illnesses, in which a person experiences severe disturbances in eating and exercise behaviours because of distortions in thoughts and emotions, especially those relating to body image or feelings of self-worth. People in all age groups, genders and socio-economic and cultural backgrounds can be affected by eating disorders. A person with an eating disorder can be underweight, within a healthy weight range, or overweight. There are four different types of eating disorders: anorexia nervosa, bulimia nervosa, binge eating disorder and eating disorders not otherwise specified (EDNOS) (MHFA, 2012).

Eating disorders are serious illnesses that often are not detected by health care professionals. An eating disorder is marked by extremes. It is present when a person experiences severe disturbances in eating behavior, such as extreme reduction of food intake or extreme overeating, or feelings of extreme distress or concern about body weight or shape. A person with an eating disorder may have started out just eating smaller or larger amounts of food than usual, but at some point, the urge to eat less or more spirals out of control. Eating disorders are very complex, and despite scientific research to understand them, the biological, behavioral and social underpinnings of these illnesses remain elusive (Mental Health First Aid, 2012).

Current trends in computing present users with the opportunity of interacting with programs to get medical direction or advice. This is well based on the concept of fuzzy logic which is a form of many-valued logic or probabilistic logic; it deals with reasoning that is approximate rather than fixed and exact. Compared to traditional binary sets (where variables may take on true or false values) fuzzy logic variables may have a truth value that ranges in degree between 0 and 1. Fuzzy logic has been extended to handle the concept of partial truth, where the truth value may range between completely true and completely false. There is a problem of detecting eating disorders in variety of persons because the symptoms are not easily detected by suffering persons due to lack of directed diagnosis.

\section{Objectives of the Study}

i. To explain eating disorder and expose its symptoms

ii. To design and implement a web based fuzzy logic system for the detection of eating disorders

\section{Rationale of the Study}

The use of computers in detecting health problems is a field of study that is important due to its reduction of workload on the part of humans and aids speed in terms of processing. This study focused on four 
types of eating disorder and the system was developed to detect these four types - anorexia nervosa, bulimia nervosa, binge eating disorder and 'eating disorders not otherwise specified' (EDNOS).

\section{Types of Eating Disorders}

Mental Health First Aid (2012) reported four different types of eating disorders: anorexia nervosa, bulimia nervosa, binge eating disorder and 'eating disorders not otherwise specified' (EDNOS). If a person one is helping is underweight and using extreme weight-loss strategies, they may have anorexia. If a person is engaging in binge eating followed by extreme weight-loss strategies, they may have bulimia. Although by definition, a person with anorexia is underweight, a person with bulimia can be slightly underweight, within a healthy weight range, or overweight. If a person regularly eats an unusually large amount of food in a short period of time, accompanied by a sense of loss of control over their eating, but does not use extreme weight-loss strategies to compensate, they may have binge eating disorder. People with binge eating disorder may be within a healthy weight range or overweight. If a person does not fit the description of anorexia, bulimia or binge eating disorder, but their attitude to food, weight, body size or shape is seriously interfering with their life, they may have EDNOS. Some examples of EDNOS include when a person is using extreme weight-loss strategies but does not have the very low body weight of a person with anorexia, or when the person has infrequent episodes of binge eating or vomiting.

\section{Anorexia Nervosa}

NIMH (2007) discussed anorexia nervosa eating disorder as characterized by emaciation, a relentless pursuit of thinness and unwillingness to maintain a normal or healthy weight, a distortion of body image and intense fear of gaining weight, a lack of menstruation among girls and women, and extremely disturbed eating behavior. Some people with anorexia lose weight by dieting and exercising excessively; others lose weight by self-induced vomiting, or misusing laxatives, diuretics or enemas. Many people with anorexia see themselves as overweight, even when they are starved or are clearly malnourished. Eating, food and weight control become obsessions. A person with anorexia typically weighs herself/himself repeatedly, portions food carefully, and eats only very small quantities of only certain foods. Some who have anorexia recover with treatment after only one episode. Others get well but have relapses. Still others have a more chronic form of anorexia, in which their health deteriorates over many years as they battle the illness.

\section{Bulimia Nervosa}

Kelly and Kathy (2008) explained that individuals with bulimia nervosa eat excessive quantities of food, then purge their bodies of the food and calories they fear by using laxatives, enemas, or diuretics, vomiting and/or exercising. Often acting in secrecy, they feel disgusted and ashamed as they binge, yet relieved of tension and negative emotions once their stomachs are empty again. Bulimia nervosa is characterized by recurrent and frequent episodes of eating unusually large amounts of food (e.g., binge-eating), and feeling a lack of control over the eating. This binge-eating is followed by a type of behavior that compensates for the binge, such as purging (e.g., vomiting, excessive use of laxatives or diuretics), fasting and/or excessive exercise. Unlike anorexia, people with bulimia can fall within the normal range for their age and weight. But like people with anorexia, they often fear gaining weight, want desperately to lose weight, and are intensely unhappy with their body size and shape. Usually, bulimic behavior is done secretly, because it is often accompanied by feelings of disgust or shame. The binging and purging cycle usually repeats several times a week. Similar to anorexia, people with bulimia often have coexisting psychological illnesses, such as depression, anxiety and/or substance abuse problems. Many physical conditions result from the purging aspect of the illness, including electrolyte imbalances, gastrointestinal problems, and oral and tooth-related problems (NIMH, 2007).

\section{Binge-Eating Disorder}

According to Jorm (2010), Binge-eating disorder is characterized by recurrent binge-eating episodes during which a person feels a loss of control over his or her eating. Unlike bulimia, binge-eating episodes are not followed by purging, excessive exercise or fasting. As a result, people with binge-eating disorder often are overweight or obese. They also experience guilt, shame and/or distress about thebingeeating, which can lead to more binge-eating. Obese people with binge-eating disorder often have coexisting psychological illnesses including anxiety, depression, and personality disorders. In addition, links between obesity and cardiovascular disease and hypertension are well documented.

Kelly (2008) noted that like people with bulimia, those with binge eating disorder experience frequent episodes of out-ofcontrol eating. The difference is that binge eaters don't purge their bodies of excess calories. According to $\mathrm{MOH}$ (2000), in sharp contrast to the undereatingbehaviour of individuals with anorexia nervosa,

people with binge eating disorder are chronic overeaters who go through long periods of frequent bouts of binge eating. These periods of overeating, often six months or longer, are followed by feelings of guilt and 
shame. Unlike people with bulimia nervosa, however, people with binge eating disorder do not engage in purging or other compensatory behaviours. Sometimes called "compulsive eating" or "food addiction," binge eating disorder is much more widespread than either bulimia or anorexia. Males, people from lower socioeconomic backgrounds and people of a wide range of ages are just as likely as middle class, adolescent females to develop this disorder. Binge eating disorder is a difficult disorder to identify. People with binge eating disorder are not always overweight, and people who are overweight are not necessarily suffering from binge eating disorder. Excessive weight gain has a myriad of causes.

\section{Eating Disorders Not Otherwise Specified (Ednos)}

A typical eating disorders (eating disorders not otherwise specified; EDNOS) including binge eating disorder. A number of people suffer from eating disorders that closely resemble anorexia nervosa and bulimia nervosa, but which are considered atypical, as they do not meet the precise diagnostic criteria for these conditions (Fairburn \& Harrison, 2003; Turner \& Bryant- Waugh, 2003; Ricca et al., 2001). In Europe, these are often termed 'atypical eating disorders' (Fairburn \& Harrison, 2003), the equivalent American term being 'eating disorders not otherwise specified' (American Psychiatric Association, 1994). For example, the patient's weight might be just above the diagnostic threshold for anorexia nervosa or she might still be menstruating. Binge eating and purging may occur less frequently than specified for a diagnosis of bulimia nervosa. Over concern with weight and shape is generally present in these disorders, although in some the primary focus is on maintaining strict control over eating. Although the diagnostic criteria may not be met, many atypical eating disorders are as severe and long lasting as anorexia nervosa and bulimia nervosa. Patients' treatment needs and prognosis may be virtually identical. Binge eating disorder (BED) is a recently described condition, first defined as a research category in DSM-IV (APA, 1994), though there is some overlap with the ICD 10 category 'Overeating associated with other psychiatric disturbance' (F50.4) (WHO, 1992). In BED, individuals engage in uncontrollable episodes of binge eating but do not use compensatory purging behaviours. These binge eating episodes are associated with three or more of the following:

i. eating much more rapidly than normal

ii. eating until feeling uncomfortably full

iii. eating large amounts of food when not physically hungry

iv. eating alone through embarrassment at the amount one is eating

v. feeling disgust or extreme guilt after over-eating.

Marked distress regarding binge eating is present and social avoidance is common.

\section{Vicious Cycle Of Eating Disorder - Self Abuse}

MOE (2000) explained that in the broadest sense, the term "eating disorder" can refer to any destructive or self-defeating pattern of eating behaviour. Typically, students with eating disorders are preoccupied with food, weight and their personal body image. It is important, however, to distinguish eating disorders from other eating-related problems. Eating disorders are complex and their causes are not well understood. A number of factors contribute to the onset of eating disorders: genetics, family relationships, trauma and individual cognitive styles. Since eating disorders most often arise during adolescence, there may be some developmental triggers as well. In general, students with eating disorders experience depression or low self-esteem and relate these problems to their body image. Disordered eating may be seen as an attempt to create, or regain, a feeling of control when the rest of life seems out of control. Often for these individuals eating, or not eating, is how they attempt to communicate their needs and to cope with high stress levels. This behaviour is supported and maintained by the individual's skewed system of logic. For many, it becomes a vicious circle, as the physical consequences of malnutrition or overeating undermine their already fragile selfconcept. One of the most life-threatening eating disorders, anorexia nervosa, can be illustrated as shown on Fig. 1 .The potential for escalation into a cycle of self-abuse is common to all eating disorders due to the effect on perception, thinking, mood and behaviour. Eating behaviours may move along a continuum of severity, from normal eating to the actual onset of bulimia or anorexia. Recent research indicates that as the disturbance in normal eating patterns becomes more extreme, so does the individual's sense of body dissatisfaction, interpersonal distrust, fear of maturing and inability to regulate impulsive behaviour. The person with an eating disorder develops patterns of self-control or self-denial that are extreme. It is also important, however, to know that an individual does not have to progress through all or any eating styles prior to developing an eating disorder. 


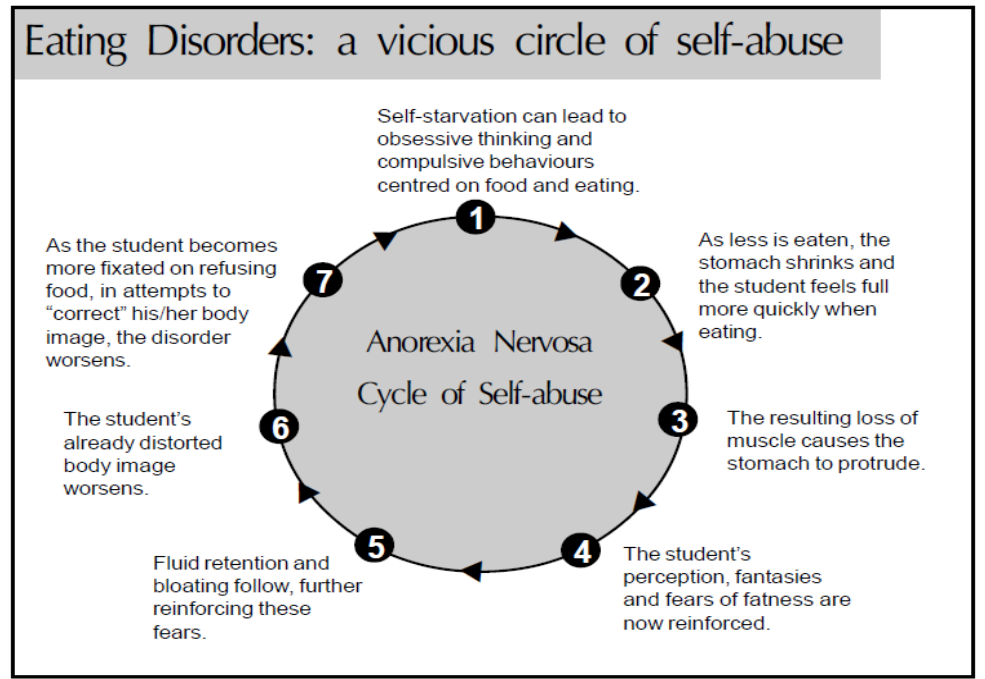

Fig. 1: Eating Disorders: a vicious circle of self - abuse

From "Eating disorders: a continuum," by J. Marchuk, notes from a presentation tothe Alberta Teachers' Association Guidance Specialist Council, Annual Conference,Banff, Alberta, November 6-8, 1997.

\section{Symptoms Of Eating Disorder}

Red flags that may indicate eating disorder include:

i. Skipping meals

ii. Making excuses for not eating

iii. Eating only a few certain "safe" foods, usually those low in fat and calories

iv. Adopting rigid meal or eating rituals, such as cutting food into tiny pieces or spitting food out after chewing

v. Cooking elaborate meals for others, but refusing to eat them themselves

vi. Collecting recipes

vii. Withdrawing from normal social activities

viii. Persistent worry or complaining about being fat

ix. A distorted body image, such as complaining about being fat despite being underweight

x. Not wanting to eat in public

xi. Frequent checking in the mirror for perceived flaws

xii. Wearing baggy or layered clothing

xiii. Repeatedly eating large amounts of sweet or high-fat foods

xiv. Use of syrup of ipecac, laxatives, the over-the-counter weight-loss drug orlistat (Alli), or over-thecounter drugs that can cause fluid loss, such as menstrual symptom relief medications

$\mathrm{xv}$. Use of dietary supplements or herbal products for weight loss

xvi. Food hoarding

xvii. Leaving during meals to use the toilet

xviii. Eating in secret

xix. The following sections discuss specific symptoms for the compulsive overeating/binge eating disorder and symptoms for anorexia/bulimia of eating disorder which share similar characteristics.

\section{Symptoms For Anorexia/Bulimia}

i. Dramatic weight loss in a relatively short period of time.

ii. Wearing big or baggy clothes or dressing in layers to hide body shape and/or weight loss.

iii. Obsession with weight and complaining of weight problems (even if "average" weight or thin).

iv. Obsession with calories and fat content of foods.

v. Obsession with continuous exercise.

vi. Frequent trips to the bathroom immediately following meals (sometimes accompanied with water running in the bathroom for a long period of time to hide the sound of vomiting).

vii. Visible food restriction and self-starvation.

viii. Visible bingeing and/or purging.

ix. Use or hiding use of diet pills, laxatives, ipecac syrup (can cause immediate death!) or enemas.

x. Isolation. Fear of eating around and with others. 
xi. Unusual Food rituals such as shifting the food around on the plate to look eaten; cutting food into tiny pieces; making sure the fork avoids contact with the lips (using teeth to scrap food off the fork or spoon); chewing food and spitting it out, but not swallowing; dropping food into napkin on lap to later throw away.

xii. Hiding food in strange places (closets, cabinets, suitcases, under the bed) to avoid eating (Anorexia) or to eat at a later time (Bulimia).

xiii. Flushing uneaten food down the toilet (can cause sewage problems).

xiv. Vague or secretive eating patterns.

xv. Keeping a "food diary" or lists that consists of food and/or behaviors (ie., purging, restricting, calories consumed, exercise, etc.)

xvi. Pre-occupied thoughts of food, weight and cooking.

xvii. Visiting websites that promote unhealthy ways to lose weight.

xviii. Reading books about weight loss and eating disorders.

xix. Self-defeating statements after food consumption.

xx. Hair loss. Pale or "grey" appearance to the skin.

xxi. Dizziness and headaches.

xxii. Frequent sore throats and/or swollen glands.

xxiii. Low self-esteem. Feeling worthless. Often putting them down and complaining of being "too stupid" or "too fat" and saying they don't matter. Need for acceptance and approval from others.

xxiv. Complaints of often feeling cold.

xxv. Low blood pressure.

xxvi. Loss of menstrual cycle.

xxvii. Constipation or incontinence.

xxviii. Bruised or calluses knuckles; bloodshot or bleeding in the eyes; light bruising under the eyes and on the cheeks.

xxix. Perfectionistic personality.

xxx. Loss of sexual desire or promiscuous relations.

xxxi. Mood swings. Depression. Fatigue.

xxxii. Insomnia. Poor sleeping habits

\section{Symptoms For Compulsive Overeating/Binge Eating Disorder}

i. Fear of not being able to control eating, and while eating, not being able to stop.

ii. Isolation. Fear of eating around and with others.

iii. Chronic dieting on a variety of popular diet plans.

iv. Holding the belief that life will be better if they can lose weight.

v. Hiding food in strange places (closets, cabinets, suitcases, under the bed) to eat at a later time.

vi. Vague or secretive eating patterns.

vii. Self-defeating statements after food consumption.

viii. Blames failure in social and professional community on weight.

ix. Holding the belief that food is their only friend.

$x$. Frequently out of breath after relatively light activities.

xi. Excessive sweating and shortness of breath.

xii. High blood pressure and/or cholesterol.

xiii. Leg and joint pain.

xiv. Weight gain.

$\mathrm{xv}$. Decreased mobility due to weight gain.

xvi. Loss of sexual desire or promiscuous relations.

xvii. Mood swings. Depression. Fatigue.

xviii. Insomnia. Poor Sleeping Habits.

\section{Contributing Factors For Eating Disorder}

According to Kristy (2000), Researchers have tried for many years to identify the causes of eating disorders. Lately, scientists have turned away from the notion of a single cause and have come to understand that a number of factors predispose people to eating disorders. Those factors include a number of personality and environmental triggers. Many individuals with eating disorders set the unattainable standards of a perfectionist for themselves, suffer from low self-esteem, and tend to develop depression. These individuals may have experienced an event threatening to their self-esteem, such as a family divorce, a change of school or loss of a friend, in the months leading to the onset of the problem. There is evidence that sexual abuse may be a factor in some cases. 
Transition is thought to be significant. The move from childhood to adolescence is closely linked to the development of eating disorders. Some researchers believe that the eating disorder may represent an attempt to delay or postpone physical and emotional maturation and the responsibility that comes with growing up. Finally, scientists believe that genetics may play a significant role, although research in this area is only in its earliest stages. Environment can be a key factor. Households that place a major stress on academic and athletic achievement are described as being more likely to inadvertently foster eating disorders. Youth drawn to highly competitive activities that focus on thinness, such as gymnastics, ballet or modeling, are also at increased risk. Second aspects of environment, cultural influences, are also thought to be a factor. We live in a culture that values thinness, especially for women. Television and print media portray desirable and successful people with sleek, even gaunt, supermodel shapes, setting an unrealistic standard for beauty. In this light, one can easily see how impressionable and vulnerable pre-adolescent and adolescents, in the midst of the physical changes of puberty, may struggle to attain these unrealistic standards and, in doing so, seriously compromise their health and wellbeing.

\section{Fuzzy Logic And Detection Of Eating Disorder}

Fuzzy logic is a multi-valued (as opposed the binary) logic developed to deal with imprecise or vague data. Classical logic holds that everything can be expressed in binary terms: 0 or 1; black or white; yes or no; in terms of Boolean algebra, everything is in one set or another but not in both. It can be described as a problemsolving control system methodology that lend itself to implementation in system raging from simple, small embedded micro controllers to large, networked, multi-channel PC or workstation acquisition and control system. Fuzzy logic is derived from Fuzzy set theory dealing with reasoning that is approximately rather than precisely deduced from classical predicate logic. It can be implemented in hardware software or a combination of both. Fuzzy logic provides a simple way to arrive at a definite conclusion based upon vague ambiguous, imprecise, noisy, or missing input information. Fuzzy logic's approach to control problem mimic how a person will make a decision in only much faster. The concept of fuzzy logic (FL) was conceived by loftizadeh, a professor at university of California at Berkley and presented not as a control methodology, as a way of processing data by allowing partial set membership rather than crisp set member or non-membership. This approach to set theory was not applied to control systems until the 70's due to insufficient small computer capability prior to the time professor Zadeh reasoned that people do not require precise, numerical information input, and yet they are capable of highly adaptive control. If feedback controllers could be programmed to accept noisy, imprecise input, they would be much more effective and perhaps easier to implement. Unfortunately, U.S manufacturers have not been so quick embrace this technology while the Europeans and Japanese have been aggressively building real products around it. Lotfi finally posited the notion of an infinite valued logic, which he introduced in his seminal work 'Fuzzy set' in 1963. This he described (the mathematics of fuzzy set theory) by extension of fuzzy logic. This theory he proposed making the membership function (or the value $\mathrm{F}$ and $\mathrm{T}$ ) operate over the range of real numbers $(0,1)$. From his perceptive, Fuzzy sets introduced as a means of representing and manipulating data that was not precise, but rather fuzzy (not expressed clearly). Fuzzy logic provides an inference morphology that enables approximate human reasoning capabilities to be applied to knowledge based system. Fuzzy logic: a logic that is used to try to make the computers behave like the human brain. The theory of fuzzy logic provides the mathematical strength to capture the uncertainties associated with human cognitive processes, such as motivated in large measure by the uncertainty and lexical imprecision being fully aware that the conventional approaches to knowledge representation lack the means of representing the meaning of fuzzy concepts.

Fuzzy logic is a logic represented by a fuzzy expression (formula) which satisfies the following:

i. $\quad$ Truth values, 0 and 1 , and variable $\mathrm{Xi}(=[0,1] . \mathrm{i}=1,2, \ldots, \mathrm{n}]$

ii. If $\mathrm{f}$ is a fuzzy expressions. $\mathrm{f}$ (not $\mathrm{f}$ ) is also a fuzzy expression.

iii If $\mathrm{f}$ and $\mathrm{g}$ are fuzzy expressions, $\mathrm{f} \Lambda \mathrm{g}$ and $\mathrm{fV} g$ are also fuzzy expressions.

A logic based on the two truth values True and False is sometimes inadequate when describing human reasoning. Fuzzy logic uses the whole interval between 0 (False) and 1 (True) to describe human reasoning.

\section{Features of Fuzzy Logic}

Emphasized in this research, can be related to the followings:

(a) In fuzzy logic, exact reasoning is viewed as limiting case of approximate reasoning.

(b) Any logical system can be fuzzified.

(c) Knowledge is interpreted as a collection of elastic or equivalently in fuzzy logic, fuzzy constrains on collection of variable.

(d) In fuzzy logic, everything is a matter of degree. However, the two main characteristics of fuzzy system that gives them better performance for specific applications are stated below: 
(1) Fuzzy logic is suitable for uncertain or approximate reasoning, especially for system with a mathematical model that is difficult to derive.

(2) Fuzzy logic allows decision making with estimated values under incomplete or uncertain information.

Conversely, fuzzy systems are methodologies to represent and process linguistic information, with mechanisms to deal with uncertainties and imprecision by means of genetic algorithms.

With such remarkable attributes, fuzzy systems have been widely successfully applied, to control, classify and model problems.

One of the most important tasks in the development of fuzzy systems is the design of its knowledge base. An expressive effort has been devised lately to develop or adapt methodologies that are capable of automatically extracting the knowledge base from numerical data. Particularly, in the framework of soft computing, significant methodologies have been proposed with the objectives of building fuzzy systems by means of genetic algorithms (Gas).

Genetic algorithms have demonstrated to be a powerful tool to perform tasks such as generation of fuzzy rule-base, optimization of fuzzy rule-base, generation of membership functions and tuning of members functions. All these tasks can be considered as optimization or search processes. Fuzzy systems generated or adapted by genetic algorithms are called genetic fuzzy system. The first step in designing a genetic fuzzy system is to decide which genetic algorithms are called genetic fuzzy system [condon et al., 200lb]. The first step in designing a genetic fuzzy system is to decide which parts of the knowledge-base (KB) are subject to optimization by the GA. The KB of a fuzzy system does not constitute a homogenous structure but is rather the union of qualitatively different components. An example, the KB of a descriptive madman type fuzzy system (i.e. the linguistic fuzzy modeling that is focused on interpretability) and is comprised of two components: a data base-base (DB), containing the definitions of the scaling factors and the membership function of the fuzzy sets associated with the linguistic labels, and a rule base (RB), constituted by the collection of fuzzy rules.

Genetic learning of fuzzy rule-base assumes a pre-defined set of fuzzy membership functions in the DB to which the rules refer to by means of linguistic labels.

\section{REASONS FOR THE USE OF FUZZY LOGIC}

Fuzzy logic offers several unique features that make it a particularly good choice for many control problems [kaehler 1996]:

(a) It is inherently robust since it does not required precise noise-free input and can be programmed to fall safely if a feedback sensor quits or it destroyed. The output control is a smooth control function despite a wide range of input

(b) Since the FL controller processes user defined governing the control system, it can be modified and tweaked easily to improve or drastically alter the system performance. New sensors can easily be incorporated into the system simply by generating appropriate governing rules.

(c) Because of the RB operation, any reasonable number of input can be processed (1-8 or more) and numerous outputs $(1-4$ or more) generated, although defining the RB quickly becomes complex if too many inputs and outputs are chosen for a single implementation since rules defining their inter-relations must also be defined. I would be better to break the control system into smaller chunks and use several smaller FL controllers distributed on the systems, each with more limited responsibilities.

(d) FL can control non-linear systems that would be difficult or impossible mathematically. This opens doors for control systems that would normally be deemed unfeasible for automation.

\section{How Fuzzy Logic Is Used}

FL requires some numerical parameters in order to operate, such as: as what is considered significant error and significant rate-of-change of error, but exact values of these numbers are usually not critical unless very responsive performance is required in which case empirical tuning would determine them. The first fuzzy controller was exhibited at Second IFSA Congress in 1987.

This controller originated from Omron Corp., a Japanese company which began research in fuzzy logic in 1984 and has since applied for over 700 patents.

Also in 1987, the Sendai Subway Automatic Train Operations Controller, designed by the Hitachi team, started operating in Sendai, Japan. The fuzzy logic in this subway system makes the journey more comfortable with smooth braking and acceleration. In 1989, Omron Corp. demonstrated fuzzy workstations at the Business Show in Harumi, Japan. Such a workstation is just a RISC-based computer, equipped with a fuzzy inference board. This fuzzy inference board is used to store and retrieve fuzzy information, and to make fuzzy inferences. For the fact that fuzzy logic can be programmed to control a varieties of input data. In this research, we used fuzzy parameters to detect the intensity of skin disease ranging from 0.0 to 1.0 .

The basic steps on how to use fuzzy logic are stated below: 
a. Define the control objectives and criteria.

b. What am I trying to control?

c. What do I have to do to control the system?

d. What kind of response do I need?

e. What are the possible (probable) system failure nodes?

a. Determine the input and output relationships and choose a minimum number of variables for input to the FL engine (typically error and rate-of-change-of-error).

b. Using the rule base structure of FL, break the control into a series of IF X AND Y THEN Z rules that defines the desired system output response for given system input parameters that are to be processed and the number of fuzzy variables and associated with each parameter. If possible, use at least one variable and its time derivative. Although it is possible to use a single instantaneous error parameter without knowing its rate of change, this cripples systems ability to minimize overshoot for a step inputs.

c. Create FL membership functions that define the meaning (values) of input terms used in the rules.

d. Create the necessary pre- and-post processing FL routines if implementing in software, evaluate the results, and tune the rules into the FL hardware engine.

e. Test the systems, evaluate the results, and tune the rules and membership functions until satisfactory results are obtained.

\section{Justification to the Proposed System}

The proposed system will 'attempt' to resolve the constraints of the current system. The question here, though, is: "how effectively can computers aid medical diagnosis?" Computers have for decades now being employed in the medical sector, sectors ranging from electronic medical records to telemedicine (which has come to include teleconsultation, teleconferencing, teleradiology, telepathology, tele-education, telemonitoring, and even telesurgery). The successes derived from the application of computers in these fields of medicine have proven to a large extent how helpful computers can be in medicine.

Meanwhile, medical diagnosis is a complex task, and so it has not been very realistic to fully automate the process. However, with recent advances in the development of intelligent systems and more attention in Artificial Intelligence as a course in the study of Computer Science, as well as its child areas like fuzzy logic and artificial neural networks which tend to emulate the biological neural networks, successes have been recorded in this rather difficult task - electronic medical diagnosis. For example, The National Vector Borne Disease Control Programme, India (in 2009) developed a fully functional telemedical system for the diagnosis and treatment of typhoid fever, and with telemedicine, made it possible for patients in remote areas to have access to the facility.

Given the advantage of the computer in the area of being able to do the underlisted, the proposed system shall attempt to solve the existing problem of eating disorder diagnosis:

i. It can perform repetitive tasks without getting tired or being affected 'emotionally';

ii. It can embody the knowledge of human experts in a domain and with the help of special software tools, manipulate the data to solve problems and make decisions in that domain;

iii. Updating the knowledge base of the computer system can be done automatically in a few seconds, with or without the help of a human; and

iv. Processes are better formalised and defined on machines.

\section{Challenges of the Proposed System}

As with all rule-based expert systems, the proposed system may have a few challenges:

i. If the system consists of several thousand rules, it takes a powerful control program to produce any conclusions in a reasonable amount of time.

ii. As the number of rules increase, the conflict set becomes large; hence, a good conflict resolving algorithm will be required if the system is to be usable and achieve the purpose for which it was designed

However, since this system is narrowed to the diagnosis of brain cancel only, as against a wider range of illnesses, it is easier to handle these challenges better. Moreover, the application of an object-oriented paradigm in the design of the system has helped to fix the issue of conflicts set to a large extent.

It is also worthy of note that the application of fuzzy-neural network approach to the design of rule-based expert systems, if well implemented, helps to minimize the setbacks listed above.

\section{Method of the Proposed System}

In designing the proposed system, the following steps were put into use:

i. Designing a Fuzzy Neural Network (FNN) that uses fuzzy classifiers for diagnosis pruning. 
ii. Designing aTelemedical component that gives non-experts and patients alike the opportunity of communicating directly or indirectly with the health expert.

\section{Diagnosis Pruning using Fuzzy Classifiers}

Implementing fuzzy classifiers not only results in a high classification accuracy but good interpretability also. This objective is accomplished by means of a series of learning algorithms including kmeans clustering, gradient descent parameter tuning, and similarity-based model simplification. The knowledge base is practically an FNN classifier. It consists of a fuzzy inference engine and a fuzzy medical rule database. The former performs fuzzy inference with respect to the presented input feature vectors so as to provide reasonable diagnostic results; the latter contains a collection of diagnostic rules acquired from available medical databases which store a great amount of data relating to patient's records or medical history.

\section{Initial Rule Learning}

The K-means clustering algorithm is applied, which is one of the simplest algorithms in the field of pattern recognition, to acquire representative information from the given patient's records or medical history. Each of the obtained clusters stands for a prototype for a particular behaviour of the medical knowledge under consideration; that is, each cluster can be used to define a diagnostic rule. Moreover, directly projecting each cluster onto individual inputs can obtain initial membership functions for each rule.

\section{Parameter Learning}

The parameter learning algorithm is developed based on the well-known gradient descent method.

\section{Model Simplification}

The fuzzy diagnostic rules of the diagnosis system are simplified in order to enhance the interpretability of the obtained rules. Based on the set-theoretic similarity measurement derived by the wellknown determinant formula of area, the strategy consists of three steps: fuzzy set mergence, antecedent feature pruning and rule number simplification. According to these steps, we simplify the fuzzy medical rule base step by step, and meanwhile enhance its interpretability and readability. It is worth noting that the set-theoretic similarity measurement proposed in the paper is different from the conventional ones, since it unifies the procedure for computing intersection area between two fuzzy sets. Fig.1 shows the entire flowchart of the model simplification algorithm.

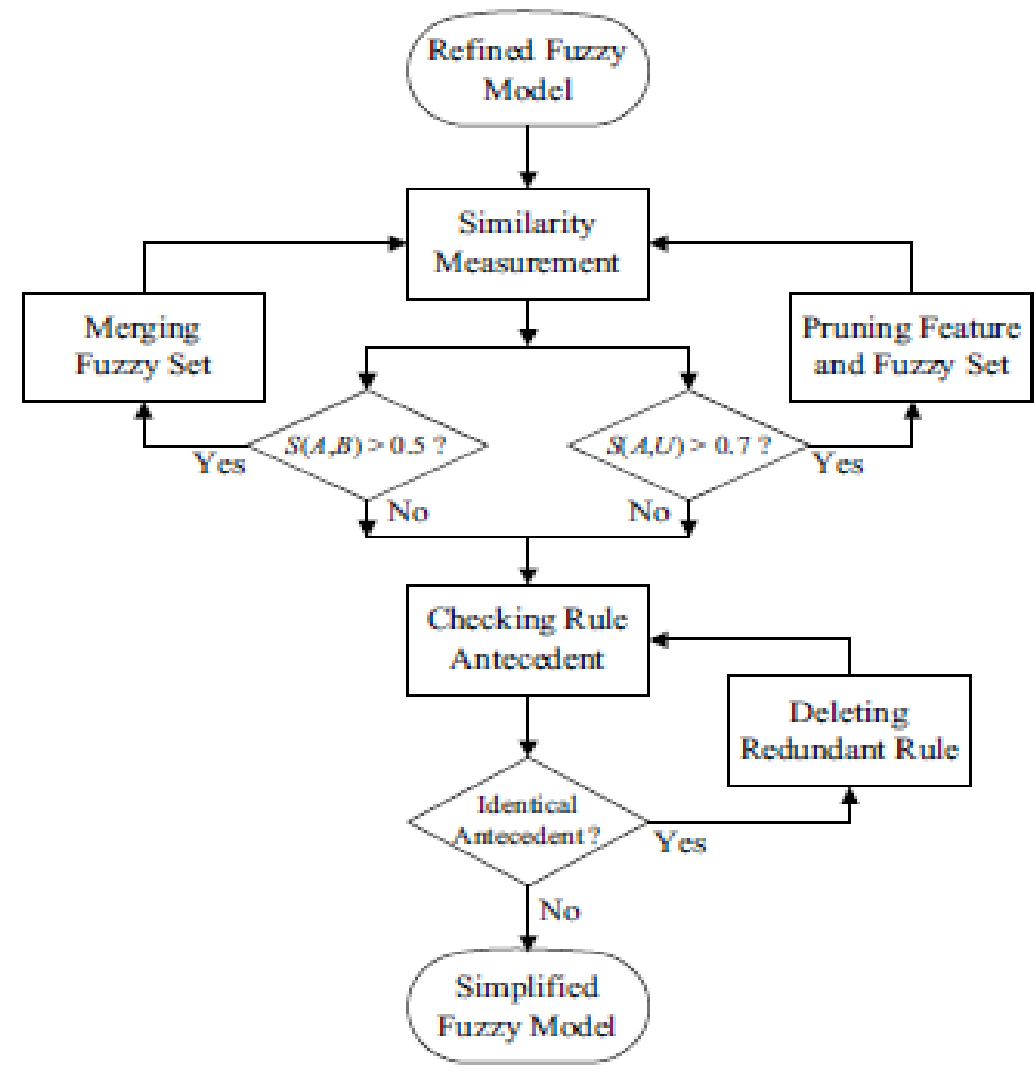

Fig. 1: Model Simplification 
The classes in the diagnosis are "Anorexia Nervosa Eating disorder", "Bulima Nervosa Eating disorder", "Binge Eating Disorder" and "eating disorders not otherwise specified (ednos)" Membership to any of these classes is determined by the pruning such that a given combination of symptoms determines which of the class the result belong to.

Table 1: Parameters for Eating disorder Detection and their associated fuzzy values

\begin{tabular}{|c|c|c|}
\hline $\mathbf{S} / \mathbf{N}$ & Parameter questions & Associated Fuzzy Range \\
\hline 1. & What is the patients' weight loss or gain? & greatly emaciated, slightly emaciated , Normal, Obese (excess fat) \\
\hline 2. & $\begin{array}{l}\text { Patient's preference to healthy weight? } \\
\text { (what patient wants) }\end{array}$ & Thin, normal, fat \\
\hline 3. & Any lack of menstruation $(\mathrm{F})$ & None, seldom, recurrent \\
\hline 4. & Does patient eat excessively? & re, excess, extreme, recurrent \\
\hline 5. & $\begin{array}{l}\text { Is patient disturbed about eating } \\
\text { excessively? }\end{array}$ & yes, detests, Not disturbed \\
\hline 6 & Any loss of control over eating? & No, mild, severe \\
\hline 7. & $\begin{array}{l}\text { Does patient use laxatives, enemas, } \\
\text { diuretics or vomiting to reduce calories }\end{array}$ & Yes, No attempt \\
\hline 8. & $\begin{array}{l}\text { Any complain of gastrointestinal } \\
\text { (stomach) problems, oral problems and } \\
\text { tooth related problems }\end{array}$ & no complain, little complain, excessive complains \\
\hline 9. & Any heart or heart related problems? & Not at all, Slight, hypertension \\
\hline 10. & Does patient eat rapidly & No, yes, extreme \\
\hline 11 & Does patient exercise regularly & No, seldom, optimal \\
\hline
\end{tabular}

The basic operation of the system is as follows. There is a universal symptom set for the eating disorder. This symptom set is as given in table 3.1 .

The patient is asked which of the symptoms he has and, where applicable, the extent to which he feels the symptom. This degree of feeling is scaled from 0 to 1 , which is fed to the k-nearest neighbour classifier. The classifier performs the job of diagnosis pruning by mapping this to a diagnosis class. The system combines the classifier which decision trees that determine with of the classes should result from the diagnosis.

\section{GRAPH}

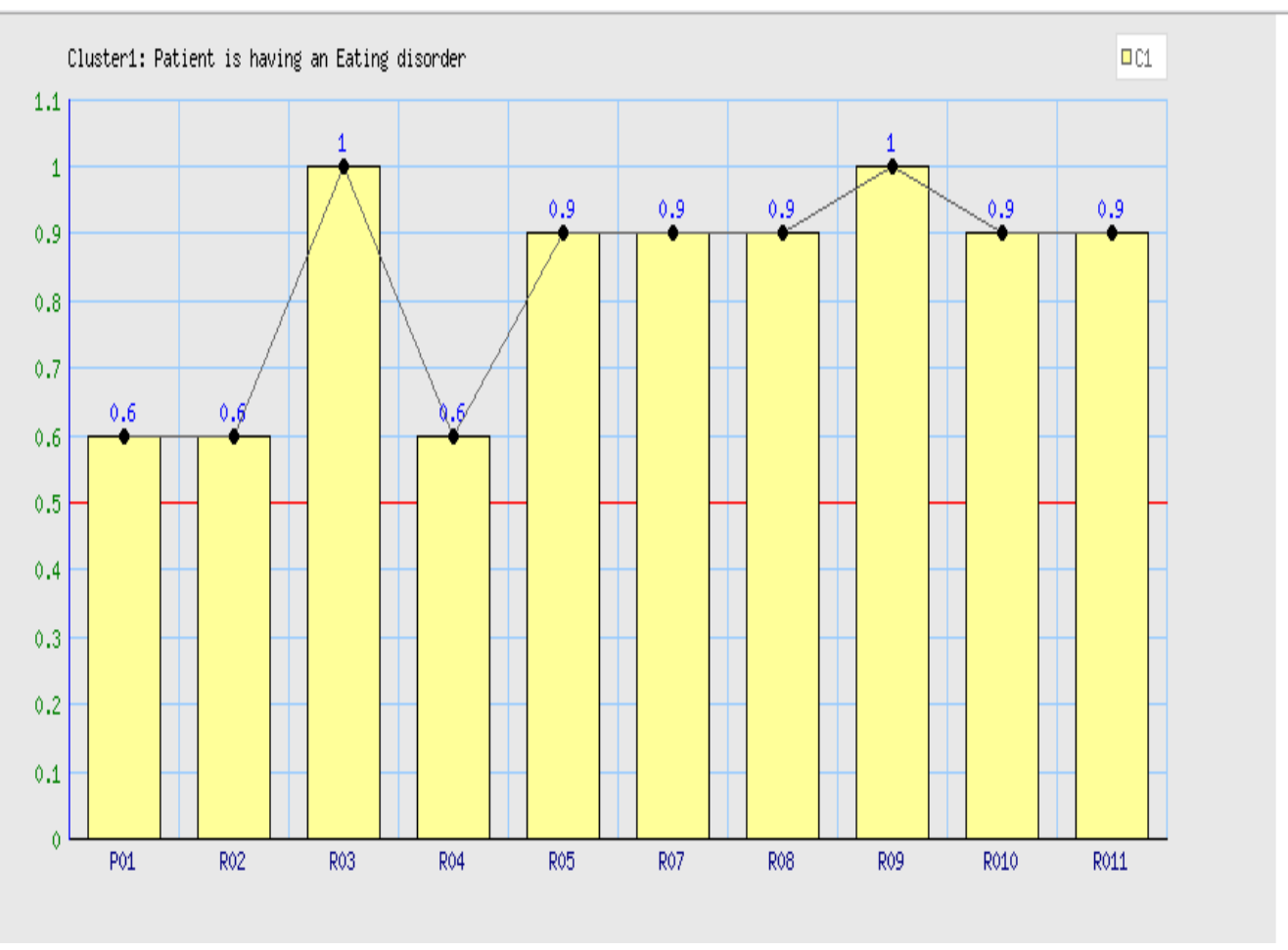

\section{The Telemedical Component}

This component makes it possible for the physician to monitor the progress of treatment for a patient. For patients in remote areas, and who do not have access to experts, diagnosis is one thing; monitoring is another. To resolve this issue of monitoring, this system shall include a component that allows patients to send 
information required by the physician, which will help the physician keep a good handle on the recovery progress of the patient in question.

Principally, one mode of doing this may be via email. The disadvantage of using email may include the fact that not all local users may have one or even know how to use it. More importantly, though, having the diagnosis component and the telemedical component in one system makes it easier for the user. The design of the telemedical component shall implement an interface that allows the user to send information required by the physician either as simple text or as an upload. Also, it shall implement automatic transmission of a diagnosis session including the patient information to the physician, so that in the event of a case that requires an emergency, the physician may act promptly and appropriately.

\section{- Eating disorder Diagnosis}

The diagnosis system is a process that involves the patient supplying answers to predefined questions as stored in the knowledge base (database) of the system. Answers supplied by the patient with respect to each of the questions are passed through a fuzzy inference engine that analyses the answers based on a fuzzy medical rule base. The existence of a medical history for a patient may help to refine the analysis carried out by the inference engine. Finally, result of the diagnosis is shown on the web-based interface. A systematic view is given by the dataflow diagram shown in fig. 2 .

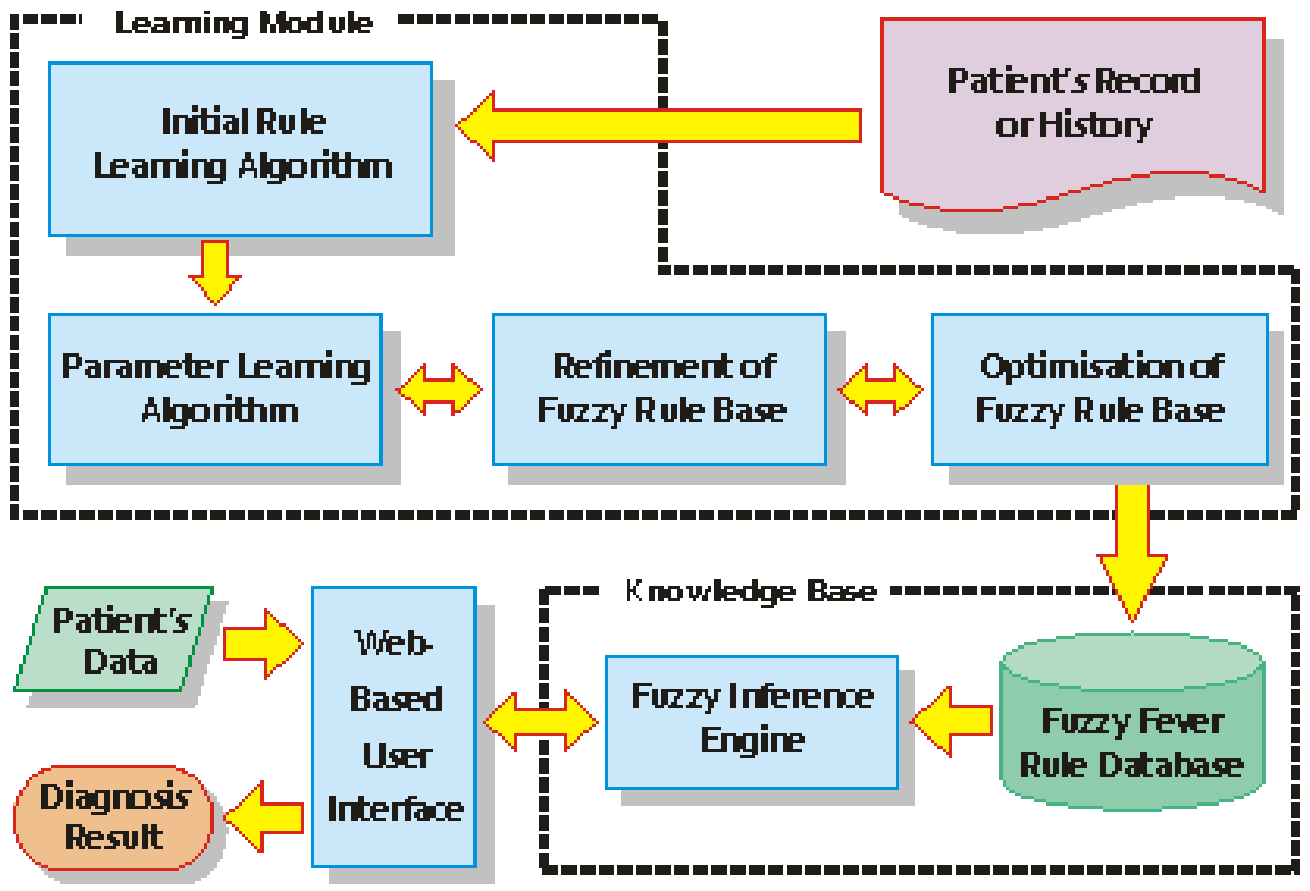

Fig. 2: The architecture of the diagnosis component 


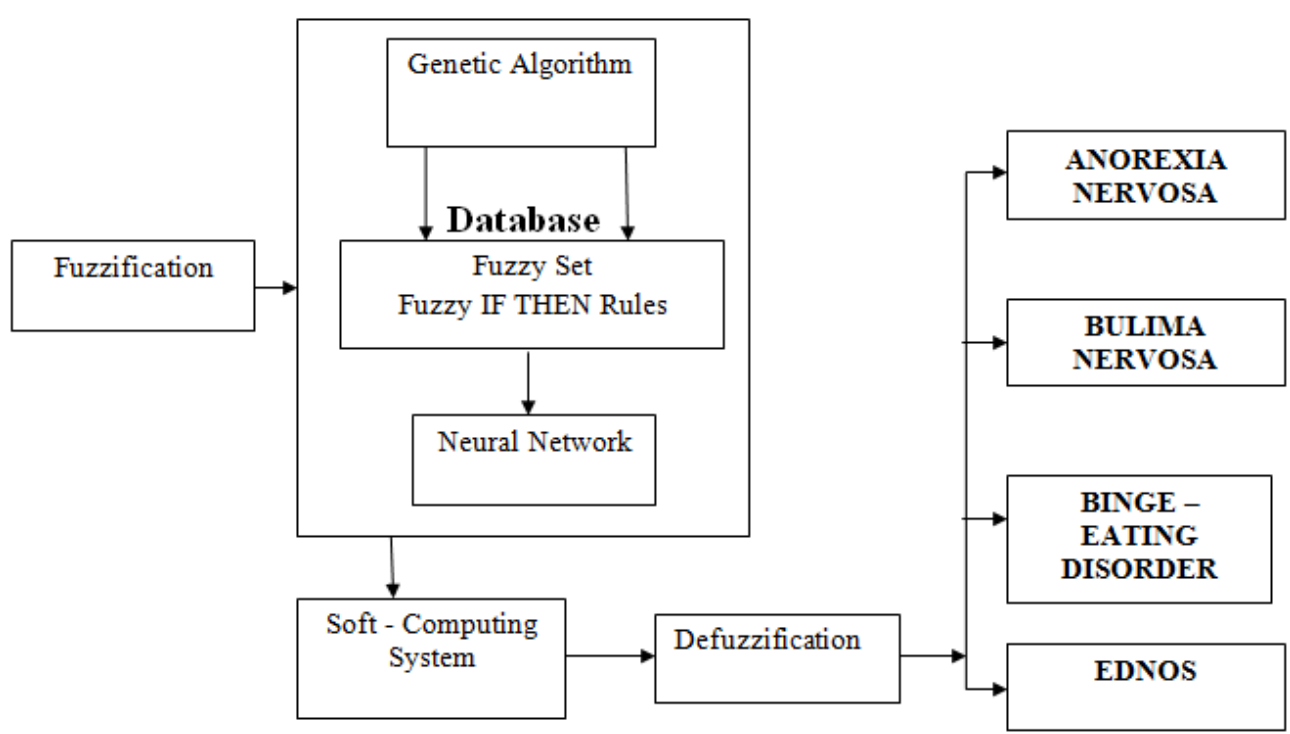

Fig. 3: Operation of the fuzzy inference engine

\section{Conclusion}

This study focused on the design and implementation of eating disorder diagnosis system for the detection of eating disorder using fuzzy logic. The system was developed to carry out the functions of managing patients'biodata, doctors' login details and patient medical history. A manual examination system is also used to serve as inputs to the system.

\section{Recommendation}

It is hereby recommended for this system to be adopted for use by hospitals and individuals for the diagnosis of patients electronically as that would ensure that patients are diagnosed promptly and it would also ease the access to data as it would be in one central location - the internet.

\section{References}

[1]. Acsm current comment (2011), "Eating disorder - A Discussion". Available online at http://www.acsm.org/docs/currentcomments/eatingdisorder.pdf

[2]. American Psychiatric Association (1994)."Work Group on Eating Disorders. Practice guideline for the treatment of patients with eating disorders (revision 2000)". American Journal ofPsychiatry, 157, 1-39.

[3]. Beglin, S.J. \& Fairburn, C.G. (1992). "What is meant by the term 'binge'?” American Journal of Psychiatry, 149(1), 123-124.

[4]. Fairburn, C.G. \& Harrison, P.J. (2003).Eating disorders. The Lancet, 361, 407-416. Available online at http://www.nimh.nih.gov/health/publications/eating-disorders/eating-disorders.pdf and http://www.somethingfishy.org/isf/signssymptoms.php

[5]. Kelly A and Kathy J (2008) "Eating Disorders- An examination of Cause and Solutions" Available online at http://www.apapracticecentral.org/outreach/eating-disorders.pdf

[6]. MHFA (2012), "Eating Disorders First Aid and Guidelines". Available online at http://www.mhfa.com.au/documents/guidelines/8244_MHFA_eatdis_guidelines_Mar09.pdfand http://www.nice.org.uk/nicemedia/pdf/CG9FullGuideline.pdf

[7]. Ricca, V., Mannucci, E., Mezzani, B. et al. (2001). Psychopathological and clinical features of outpatients with an eating disorder not otherwise specified. Eat Weight Disord, 6, 157-165.

[8]. Stanley L. Hunt (2004). "Managing common Eating Disorders:" Available online at http://www.health.gov.au/internet/main/publishing.nsf/Content/CC120D574339D02BCA25727D00104BB2/\$File/whateat.pdf

[9]. Tony Jorm (2010), "Common Eating Disorders". Available online at http://oyc.yale.edu/sites/default/files/EatingDisorders.pdf

[10]. Turner, H. \& Bryant-Waugh, R. (2003)." Eating disorder not otherwise specified (EDNOS) profiles of clients presenting at a community eating disorder service". European Eating Disorders Review. 